\title{
Seguridad de los anticoagulantes orales en enfermedad renal crónica avanzada
}

\section{Safety of oral anticoagulants in advanced chronic kidney disease}

\author{
Juliana Marcela Bustamante-Trespalacios, Maria Cristina Uribe-Barrada, \\ Susana Castro-Sánchez, Juan Carlos Arrieta-Bechara • Medellín (Colombia).
}

DOI: https://doi.org/10.36104/amc.2021.1945

\section{Resumen}

Antecedentes: los pacientes que toman anticoagulantes orales a largo plazo y además cursan con enfermedad renal crónica (ERC), tienen mayor probabilidad de tener sangrados.

Métodos: estudio de cohorte descriptivo retrospectivo en el cual se revisaron historias clínicas de pacientes anticoagulados y con ERC 3 en adelante, atendidos en una clínica de anticoagulación con el fin de evaluar eventos hemorrágicos y características basales de la población en un periodo de dos años

Resultados: se incluyeron 238 pacientes. Los anticoagulantes usados fueron warfarina (45\%), rivaroxabán (31.5\%), apixabán (14.3\%) y dabigatrán (3.4\%). Según la clasificación KDIGO 78\% de los pacientes tenían ERC G3 (37.3\% G3a y 40.7\% G3b), 15.9\% G4 y 5.8\% G5 con terapia de reemplazo renal (TRR). En el periodo de estudio solo 20 pacientes $(8.4 \%)$ tuvieron eventos hemorrágicos, de estos, 7 (35\%) fueron mayores (cuatro asociados a warfarina, dos rivaroxabán y uno apixabán). Los otros 13 sangrados fueron menores y asociados a warfarina en $46.1 \%$ de los casos. El sangrado digestivo fue el más frecuente (35\%), seguido por tejidos blandos (30\%). Sólo hubo un sangrado fatal el cual se dio en sistema nervioso central (SNC) en un paciente con ERC G4.

Conclusión: se apreció una baja tasa de sangrado, lo que podría estar relacionado con el estrecho seguimiento de una clínica de anticoagulación. El anticoagulante que más frecuentemente se asoció con sangrado fue warfarina, lo cual puede estar relacionado con un bajo tiempo en rango terapéutico (48.8\%). Por la baja tasa de eventos, no fue posible la realización de comparaciones. (Acta Med Colomb 2021; 46. DOI: https://doi.org/10.36104/amc.2021.1945).

Palabras clave: anticoagulante, seguridad, enfermedad renal crónica, sangrado mayor, sangrado menor, hemorragia, apixaban, rivaroxaban, dabigatran.

\section{Abstract}

Background: patients who take long-term oral anticoagulants and also have CKD have a greater probability of bleeding.

Methods: a retrospective, descriptive cohort study reviewing the clinical charts of anticoagulated patients with Stage 3 CKD or above seen at an anticoagulation clinic, in order to evaluate hemorrhagic events and baseline characteristics of the population over a two-year period.

Results: 238 patients were included. The anticoagulants used were warfarin (45\%), rivaroxaban $(31.5 \%)$, apixaban $(14.3 \%)$ and dabigatran (3.4\%). According to the KDIGO classification, $78 \%$ of the patients had CKD G3 $(37.3 \% \mathrm{G} 3 \mathrm{a}$ and $40.7 \% \mathrm{G} 3 \mathrm{~b}), 15.9 \% \mathrm{G} 4$ and $5.8 \%$ G5 with renal replacement therapy (RRT). During the study period, only 20 patients $(8.4 \%)$ had hemorrhagic events; of these, seven (35\%) were major (four associated with warfarin, two with rivaroxaban and one with apixaban). The other 13 bleeds were minor and associated with warfarin in $46.1 \%$ of the cases. Gastrointestinal bleeding was the most common (35\%), followed by soft tissues (30\%). There was only one fatal bleed, which occurred in the central nervous system (CNS) in a patient with CKD G4.

Conclusion: a low rate of bleeding was found, which could be related to close follow up by an anticoagulation clinic. The anticoagulant most frequently associated with bleeding was warfarin,
Dras. Juliana Marcela Bustamante-Trespalacios y María Cristina Uribe-Barrada: Residentes de Medicina Interna; Susana Castro-Sánchez: Estudiante de Medicina; Dr. Juan Carlos ArrietaBechara: Médico Internista y Vascular. Profesor de Medicina Vascular. Director Laboratorio Vascular y Director Clínica de Anticoagulación de la IPS Universitaria Universidad de Antioquia. Universidad de Antioquia. Medellín (Colombia).

Correspondencia: Dra. María Cristina UribeBarrada. Medellín (Colombia).

E-Mail: crysuriba@gmail.com

Recibido: 12/VII/2020 Aceptado: 01/III/2021 
which could be related to a low time in therapeutic range $(48.8 \%)$. Due to the low rate of events, comparisons could not be made. (Acta Med Colomb 2021; 46. DOI: https://doi.org/10.36104/ amc.2021.1945).

Key words: anticoagulants, chronic renal failure, major bleeding, minor bleeding, safety, hemorrhage, warfarin, apixaban, rivaroxaban, dabigatran .

\section{Introducción}

Los anticoagulantes orales directos (DOAC, por sus siglas en inglés) surgieron en respuesta a la búsqueda de un anticoagulante ideal, después que la warfarina fuera por mucho tiempo el único disponible $(1,2)$. Los DOAC como rivaroxabán, apixabán, dabigatrán, edoxabán y betrixabán (los tres primeros disponibles en el mercado colombiano), son recomendados ampliamente por demostrar eficacia y mayor seguridad, al compararse en pacientes con fibrilación auricular no valvular (FA) (3-6).

La warfarina es un medicamento directamente relacionado con eventos adversos hemorrágicos, cuya frecuencia se estima en $3 \%$ a tres meses para sangrado mayor y fatal (7), lo cual en nuestro medio podría ser mayor debido a la falta de adherencia al manejo, la dificultad en la educación de los pacientes, la irregularidad en el seguimiento y el bajo porcentaje de tiempo en rango terapéutico (TRT) (2, 8). Opuesto a esto, se ha evidenciado que los DOAC tienen ventajas sobre la warfarina como el inicio rápido de acción, efecto predecible, poca variabilidad interindividual, pocas interacciones medicamentosas, mejoría de la adherencia, disminución de costos por hospitalizaciones y el no requerimiento de monitorización periódica y ajustes frecuentes en las dosis; además de la reducción de sangrado mayor como desenlace fundamental de seguridad $(9,10)$.

Desde la farmacocinética se conoce que dichos medicamentos difieren sustancialmente en cuanto a biodisponibilidad oral, unión a proteínas plasmáticas y eliminación renal $(11,12)$. Ésta última es más alta para el dabigatrán $(80 \%)$, mientras que edoxabán, rivaroxabán, apixabán y betrixabán se excretan sin cambios en 50, 33, 27 y $11 \%$ de la dosis, respectivamente $(3,13-15)$.

Es frecuente encontrar pacientes con indicación de anticoagulación en la práctica médica diaria, dada principalmente por fibrilación auricular (FA), además muchos de éstos tienen enfermedad renal crónica (ERC) $(16,17)$, lo que obliga a evaluar si la terapia convencional es igualmente válida para esta población específica.

Los grandes ensayos aleatorios que han evaluado la efectividad y la seguridad de los anticoagulantes directos vs warfarina, en general, excluyeron pacientes con ERC avanzada y en diálisis, por lo que actualmente su manejo es controversial (18-21). Hasta el momento, sólo se tienen estudios observacionales (22-24) con tamaño de muestra pequeño y estudios con bases farmacológicas como único soporte para tomar decisiones médicas complejas, pero aún no se sabe si son las mejores para el paciente y el sistema de salud. De acuerdo con lo anterior, el objetivo de este trabajo es describir las características sociodemográficas, clínicas y de laboratorio de los sujetos anticoagulados y con enfermedad renal crónica avanzada en estadios 3, 4 y 5 de la IPS Universitaria Universidad de Antioqia, en la ciudad de Medellín (Colombia); además describir los casos de hemorragia menor, mayor y fatal presentados.

\section{Diseño del estudio}

\section{Material y métodos}

Estudio de cohorte descriptivo retrospectivo sobre la seguridad de los anticoagulantes en pacientes con enfermedad renal crónica en estadios 3, 4 y 5.

\section{Lugar}

En el periodo entre enero de 2017 y diciembre de 2018 se realizó seguimiento a las historias clínicas de los pacientes anticoagulados atendidos en el programa ambulatorio de la "clínica de anticoagulación" de la institución.

\section{Participantes}

Los criterios de inclusión fueron: pacientes mayores de 18 años, con diagnóstico previo de ERC determinado por el médico tratante o que tuvieran depuración de creatinina $<60 \mathrm{~mL} / \mathrm{min} / 1.73 \mathrm{~m}^{2}$ en dos mediciones consecutivas con diferencia mínimo de tres meses y que tuvieran indicación de anticoagulación durante un tiempo mínimo de tres meses.

Se excluyeron pacientes sin diagnóstico claro o sin indicación conocida para la anticoagulación; aquellos que en el seguimiento solo tenían un valor de creatinina alterado y los pacientes en quienes no se logró un seguimiento como mínimo de dos citas con diferencia de tres meses.

\section{Variables}

Se tuvieron en cuenta para los análisis variables como: sexo, edad, régimen de seguridad social, indicación de anticoagulación, anticoagulante y en caso de tener warfarina INR en cada visita y al momento del sangrado, además el TRT; también se incluyeron estadio de ERC, comorbilidades y medicamentos previos a la anticoagulación. Sangrado mayor o menor, órgano afectado, necesidad de transfusión, TFG y comorbilidades. La clase funcional se determinó según el reporte en la historia clínica.

La ERC se define como la presencia de alteraciones en la estructura o función renal durante al menos tres meses y con implicaciones para la salud. Cualquiera de los siguientes marcadores de daño renal debe estar presente durante tres 
meses: albuminuria elevada, alteraciones en el sedimento urinario, alteraciones electrolíticas u otras alteraciones de origen tubular, alteraciones estructurales histológicas, alteraciones estructurales en pruebas de imagen, trasplante renal o tener evidencia de TFG $<60 \mathrm{~mL} / \mathrm{min} / 1.73 \mathrm{~m}^{2}$ (25). ( $\mathrm{La}$ duración mayor de tres meses de alguna de estas alteraciones se determinaba según la historia clínica).

La evaluación del estadio de la enfermedad renal se hizo mediante la determinación de la creatinina sérica con la fórmula CKD-EPI (CKD Epidemiology Collaboration), como lo recomienda la guía KDIGO publicada en el año 2012 (25) y basados en la TFG así: siendo normal (G1) TFG $>90 \mathrm{~mL} /$ $\mathrm{min} / 1.73 \mathrm{~m}^{2}, \mathrm{G} 2: 60-80 \mathrm{~mL} / \mathrm{min} / 1.73 \mathrm{~m}^{2}$, G3a: $45-59 \mathrm{~mL} /$ $\mathrm{min} / 1.73 \mathrm{~m}^{2}$, G3b: $30-44 \mathrm{~mL} / \mathrm{min} / 1.73 \mathrm{~m}^{2}$, G4: $15-29 \mathrm{~mL} /$ $\min / 1.73 \mathrm{~m}^{2}$ y G5: $<15 \mathrm{~mL} / \mathrm{min} / 1.73 \mathrm{~m}^{2}$.

Para la evaluación de la hemorragia, se utilizaron los criterios de la Sociedad Internacional de Hemostasia y Trombosis (ISTH) (26)

Sangrado mayor: sangrado que es clínicamente evidente y que satisface uno de los siguientes criterios:

- Hemorragia que resulta en una disminución de la hemoglobina de $2 \mathrm{~g} / \mathrm{dL}$ o más durante un período de 24 horas.

- Sangrado que requiere transfusión de dos o más unidades de concentrados de glóbulos rojos.

- Sangrado que ocurre en un sitio crítico (intracraneal, intraespinal, intraocular, pericárdico, intraarticular, intramuscular con síndrome compartimental o retroperitoneal).

- Sangrado que conduce a la muerte.

Sangrado menor: evento hemorrágico que es clínicamente evidente, que no satisface ninguno de los criterios previos requeridos para que el evento sea adjudicado como un evento mayor de sangrado, esto conduce a:

- Ingreso hospitalario por hemorragia.

- Tratamiento médico o quirúrgico guiado por un médico para sangrado.

- Un cambio en la terapia antitrombótica.

Sangrado fatal: se define como un evento hemorrágico que es la principal causa de muerte o contribuye directamente a la muerte.

\section{Fuente de los datos}

Base de datos de la clínica de anticoagulación e historias clínicas de la IPS Universitaria Universidad de Antioqia.

\section{Sesgos}

Por la naturaleza descriptiva del estudio, existen sesgos asociados a la falta de aleatorización de los pacientes, también está implícita la falta de representatividad de la población y ausencia de algunos datos en las historias clínicas. Como control de sesgos se evaluaron historias clínicas de diferentes especialistas en fechas aleatorias y citas de la clínica de anticoagulación.

\section{Tamaño de muestra}

Por ser un estudio descriptivo no se hizo el cálculo del tamaño de la muestra y se seleccionó, por conveniencia, a todos los pacientes atendidos en el periodo del estudio. De 1338 pacientes evaluados en la clínica de anticoagulación, se analizaron 238 historias clínicas que cumplían con los criterios de inclusión mencionados.

\section{Métodos estadísticos}

Las variables nominales se representan mediante frecuencias absolutas y relativas. Las variables continuas se presentan mediante promedios y desviaciones estándar. El único valor faltante en los datos de las historias clínicas fue la raza, la cual se asumió para todos los pacientes como "no afroamericano" para aplicar la fórmula de CKD-EPI para obtener la TFG.

Se realizó el método de Rosendaal para el cálculo del TRT.

La base de datos se elaboró empleando el programa Excel 2017 (Microsoft Corp.) y para el análisis se utilizó el software estadístico IBM SPSS Statistics 20.

El proyecto se presentó a la IPS Universitaria Universidad de Antioqia, fue analizado y aprobado por el Comité de Ética e Investigación de esta entidad. Para la elaboración de esta investigación, se tuvieron en cuenta los principios bioéticos, se obtuvieron resultados sin vulnerar los derechos de los pacientes y con el objetivo de mejorar los conocimientos sobre las patologías en pro de la población afectada y la investigación médica. Por ser un estudio de tipo descriptivo no se obtuvo consentimiento informado de los pacientes.

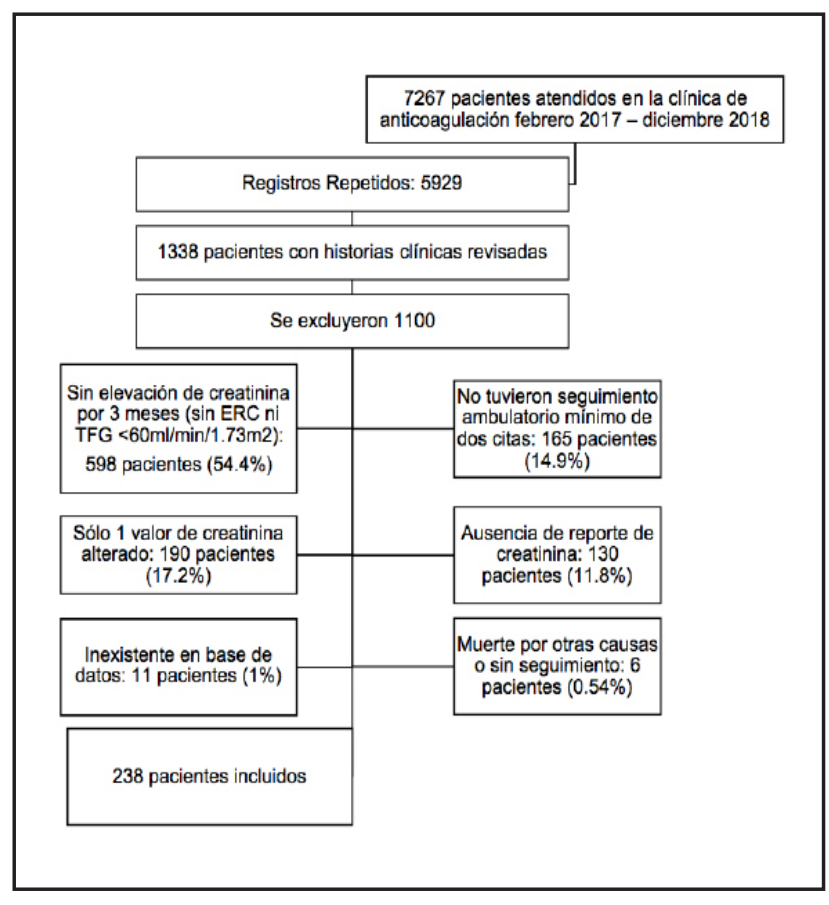

Figura 1. Diagrama de flujo que representa el proceso de selección de los participantes. 


\section{Resultados}

En la base de datos de la clínica de anticoagulación, se hicieron un total de 7267 atenciones desde enero de 2017 hasta diciembre de 2018. Luego de descartar los registros repetidos, se revisaron 1338 historias clínicas para seleccionar aquellos que cumplieron con los criterios de inclusión, para un total de 238 pacientes (Figura 1).

La principal causa de anticoagulación fue la fibrilación auricular (81.9\%), seguida de la enfermedad tromboembólica venosa tanto en miembros inferiores como pulmonar (7.6\%). El grupo de edad más frecuente fue 81-90 años (39.1\%), la distribución de sexos fue similar con 52.1\% mujeres, $47.9 \%$ hombres y en cuanto a la seguridad social, $65.5 \%$ pertenecía al régimen contributivo y $34.5 \%$ al subsidiado.

El anticoagulante más utilizado fue la warfarina (45\%), seguido de rivaroxabán (31.5\%) y apixabán (14.3\%). El total de pacientes que cambiaron de anticoagulante durante la evolución fue 36 pacientes (Tabla 1).

El promedio del TRT de los pacientes que usaban warfarina fue $48.8 \%$ y la mediana $51 \%$ (0-100\% para los valores mínimo y máximos respectivamente).

El estadio de enfermedad renal crónica en el que se encontraban la mayoría de los pacientes era G3a (37.3\%) y G3b (40.7\%) y la mayor frecuencia de sangrado se presentó en este estadio. Sólo hubo 5.8\% en G5 y de ellos, 100\% estaban en terapia de reemplazo renal (Tabla 2).

Del total de pacientes analizados se evidenció sangrado en $20(8.4 \%)$ y de éstos, 7 (35\%) tuvieron sangrado mayor. La warfarina fue el anticoagulante relacionado con $50 \%$ de estos casos, seguido de rivaroxabán (20\%) y apixabán y dabigatrán ( $15 \%$ cada uno). El sitio de sangrado mayor más frecuente fue en tracto digestivo (cinco pacientes); se presentó sólo un caso de hemorragia fatal en un paciente de 95 años, con enfermedad renal estadio 3b que recibía apixabán y presentó episodio convulsivo, trauma encefalocraneano (TEC) moderado y sangrado en sistema nervioso central (SNC). El resto de hemorragias fueron menores (13 pacientes), $46.1 \%$ fue por warfarina y $15 \%$ por dabigatrán. El sitio de sangrado más frecuente de éstos fue en tejidos blandos (30\%).

De los 10 pacientes que sangraron con el uso de warfarina: seis tenían INR supraterapéutico, tres INR en rango de 2-3 y uno INR menor de 2.0 (Tabla 3 ).

Tabla 2. Estadio de enfermedad renal crónica y ocurrencia de sangrado.

\begin{tabular}{|l|c|c|c|c|}
\hline \multirow{2}{*}{} & \multicolumn{2}{|c|}{ Total pacientes } & \multicolumn{2}{c|}{ Pacientes con sangrado } \\
\cline { 2 - 5 } & $\begin{array}{c}\text { Número } \\
(\mathbf{n = 2 3 8})\end{array}$ & $\mathbf{\%}$ & $\begin{array}{c}\text { Número } \\
(\mathbf{n = 2 0})\end{array}$ & $\mathbf{\%}$ \\
\hline E3a & 89 & 37.3 & 11 & 55.0 \\
\hline E3b & 97 & 40.7 & 4 & 20.0 \\
\hline E4 & 38 & 15.9 & 3 & 15.0 \\
\hline $\begin{array}{l}\text { E5 - terapia de } \\
\text { reemplazo renal }\end{array}$ & 14 & 5.8 & 2 & 10.0 \\
\hline
\end{tabular}

Tabla 1. Descripción de características basales de los pacientes.

\begin{tabular}{|c|c|c|c|}
\hline \multicolumn{2}{|c|}{ Características (n=238) } & \multirow{2}{*}{$\begin{array}{c}\# \\
114\end{array}$} & \multirow{2}{*}{$\begin{array}{r}\% \\
47.9\end{array}$} \\
\hline Sexo & Masculino & & \\
\hline & Femenino & 124 & 52.1 \\
\hline \multirow[t]{6}{*}{ Edad } & $40-50$ & 3 & 1.3 \\
\hline & $51-60$ & 12 & 5.0 \\
\hline & $61-70$ & 31 & 13.0 \\
\hline & $71-80$ & 78 & 32.8 \\
\hline & $81-90$ & 93 & 39.1 \\
\hline & $>91$ & 21 & 8.8 \\
\hline \multirow{2}{*}{$\begin{array}{l}\text { Seguridad } \\
\text { social }\end{array}$} & Subsidiado & 82 & 34.5 \\
\hline & Contributivo & 156 & 65.5 \\
\hline \multirow{7}{*}{$\begin{array}{l}\text { Indicaciones de } \\
\text { anticoagulación }\end{array}$} & Fibrilación auricular & 195 & 81.9 \\
\hline & Enfermedad tromboembólica venosa & 18 & 7.6 \\
\hline & Flutter atrial & 8 & 3.4 \\
\hline & Válvula cardíaca mecánica & 6 & 2.5 \\
\hline & Trombo en cavidad cardiaca & 6 & 2.5 \\
\hline & Otro & 4 & 1.7 \\
\hline & Válvula cardiaca biológica + FA & 1 & 0.4 \\
\hline \multirow[t]{6}{*}{ Anticoagulante } & Warfarina & 107 & 45.0 \\
\hline & Rivaroxabán & 75 & 31.5 \\
\hline & Apixabán & 34 & 14.3 \\
\hline & Enoxaparina + warfarina & 12 & 5.0 \\
\hline & Dabigatrán & 8 & 3.4 \\
\hline & Dalteparina + warfarina & 2 & 0.8 \\
\hline \multirow[t]{14}{*}{ Comorbilidades } & Falla cardiaca & 126 & 52.9 \\
\hline & Hipertensión arterial & 174 & 73.1 \\
\hline & Enfermedad pulmonar & 97 & 40.8 \\
\hline & Diabetes mellitus & 81 & 34.0 \\
\hline & Enfermedad tiroidea & 74 & 31.1 \\
\hline & Enfermedad coronaria & 63 & 26.5 \\
\hline & Aneurismas & 10 & 4.2 \\
\hline & Úlcera digestiva & 6 & 2.5 \\
\hline & Cirrosis & 6 & 2.5 \\
\hline & Enfermedad carotidea & 5 & 2.1 \\
\hline & Lupus eritematoso sistémico & 4 & 1.7 \\
\hline & Cáncer & 4 & 1.7 \\
\hline & Varices esofágicas & 2 & 0.8 \\
\hline & Ninguna & 2 & 0.8 \\
\hline \multirow[t]{11}{*}{ Medicamentos } & IBP & 95 & 39.9 \\
\hline & ASA & 24 & 10.1 \\
\hline & Ranitidina & 10 & 4.2 \\
\hline & Anticonvulsivantes & 7 & 2.9 \\
\hline & Amiodarona & 5 & 2.1 \\
\hline & Antibiótico & 1 & 0.4 \\
\hline & Antituberculosos & 1 & 0.4 \\
\hline & Gemfibrozilo & 1 & 0.4 \\
\hline & AINES & 0 & 0.0 \\
\hline & Ninguno & 0 & 0.0 \\
\hline & Otros & 116 & 48.7 \\
\hline
\end{tabular}


Tabla 3. Análisis de eventos hemorrágicos según tipo de hemorragia: mayor o menor.

\begin{tabular}{|c|c|c|c|c|}
\hline \multirow[b]{2}{*}{ Pacientes totales $(n=20)$} & \multicolumn{2}{|c|}{$\begin{array}{c}\text { Sangrado mayor } \\
(n=7)\end{array}$} & \multicolumn{2}{|c|}{$\begin{array}{c}\text { Sangrado } \\
\text { menor }(n=13)\end{array}$} \\
\hline & $\#$ & $\%$ & $\#$ & $\%$ \\
\hline \multicolumn{5}{|l|}{ Sexo } \\
\hline Masculino & 5 & 25.0 & 11 & 55.0 \\
\hline Femenino & 2 & 10.0 & 2 & 10.0 \\
\hline \multicolumn{5}{|l|}{ Tipo de sangrado } \\
\hline No fatal & 6 & 30.0 & 13 & 65.0 \\
\hline Fatal & 1 & 5.0 & 0 & 0.0 \\
\hline \multicolumn{5}{|l|}{ Edad (años) } \\
\hline 51 a 60 & 1 & 5.0 & 2 & 10.0 \\
\hline 61 a 70 & 1 & 5.0 & 0 & 0.0 \\
\hline 71 a 80 & 2 & 10.0 & 5 & 25.0 \\
\hline 81 a 90 & 2 & 10.0 & 4 & 20.0 \\
\hline Mayor a 90 & 1 & 5.0 & 2 & 10.0 \\
\hline \multicolumn{5}{|l|}{ Régimen } \\
\hline Contributivo & 4 & 20.0 & 10 & 50.0 \\
\hline Subsidiado & 3 & 15.0 & 3 & 15.0 \\
\hline \multicolumn{5}{|l|}{ Indicación de anticoagulación } \\
\hline Fibrilación auricular & 6 & 30.0 & 11 & 55.0 \\
\hline Enfermedad tromboembólica venosa & 0 & 0.0 & 2 & 10.0 \\
\hline Válvula cardíaca mecánica & 1 & 5.0 & 0 & 0.0 \\
\hline \multicolumn{5}{|l|}{ Anticoagulante } \\
\hline Warfarina & 4 & 20.0 & 6 & 30.0 \\
\hline INR $>3.0$ & 1 & 5.0 & 5 & 25.0 \\
\hline INR 2-3 & 2 & 10.0 & 1 & 5.0 \\
\hline INR $<2$ & 1 & 5.0 & 0 & 0.0 \\
\hline Rivaroxabán & 2 & 10.0 & 2 & 10.0 \\
\hline Apixabán & 1 & 5.0 & 2 & 10.0 \\
\hline Dabigatrán & 0 & 0.0 & 3 & 15.0 \\
\hline \multicolumn{5}{|l|}{ Estadio enfermedad renal } \\
\hline E3a & 4 & 20.0 & 7 & 35.0 \\
\hline $\mathrm{E} 3 \mathrm{~b}$ & 1 & 5.0 & 3 & 15.0 \\
\hline E4 & 1 & 5.0 & 2 & 10.0 \\
\hline E5 & 1 & 5.0 & 1 & 5.0 \\
\hline \multicolumn{5}{|l|}{ Sitio afectado } \\
\hline Gastrointestinal & 5 & 25.0 & 3 & 15.0 \\
\hline Genitourinario & 1 & 5.0 & 3 & 15.0 \\
\hline Sistema nervioso central & 1 & 5.0 & 0 & 0.0 \\
\hline Tejido blando & 0 & 0.0 & 6 & 30.0 \\
\hline Mucosa nasal - oral & 0 & 0.0 & 2 & 10.0 \\
\hline \multicolumn{5}{|l|}{ Transfusiones } \\
\hline SI & 3 & 15.0 & 0 & 0.0 \\
\hline NO & 4 & 20.0 & 13 & 65.0 \\
\hline
\end{tabular}

La caracterización de cada uno de los sujetos con sangrado se presenta en la Tabla 4.

\section{Discusión}

En este estudio se caracterizó a los pacientes con ERC en estadios 3, 4 y cinco atendidos en la clínica de anticoagulación de la IPS Universitaria Universidad de Antioqia de Medellín - Colombia en un período de dos años.

Es frecuente encontrar pacientes con indicación de anticoagulación en la práctica médica diaria, debido a la alta prevalencia de fibrilación auricular (FA) y su aumento con la edad, siendo alrededor de $2.3 \%$ a los 40 años y mayor de $6 \%$ en mayores de 65 años (27). Por otro lado, la enfermedad renal crónica (ERC) con una prevalencia alrededor de $12 \%$ en la población general (dos por cada 100 Habitantes en Colombia) para el 2014 (28), se ha establecido como factor de riesgo cardiovascular independiente, puesto que se ha relacionado fisiopatológicamente tanto con eventos trombóticos como hemorrágicos $(29,30)$.

Aproximadamente uno de cada cinco pacientes con ERC, tienen FA, lo cual es 2-3 veces mayor que en la población sin ERC (31). Para aquellos que se encuentran en diálisis peritoneal, la prevalencia es hasta $7 \%$ y si están en hemodiálisis es $13 \%$. El porcentaje de pacientes con FA y ERC aumenta también con la edad (32). Nuestro estudio evidenció que el $51.7 \%$ de los pacientes tenían edad mayor de 70 años.

Acorde con la literatura, la fibrilación auricular es la causa más frecuente de anticoagulación en pacientes con ERC, para nuestro estudio correspondió a $81.5 \%$ de los pacientes, similar a los hallazgos de reportes a nivel mundial $(33,34)$.

Respecto a la seguridad de los anticoagulantes, un estudio realizado en Canadá con 1626 pacientes en hemodiálisis que usaban warfarina arrojó un HR ajustado para sangrado de 1.44; IC de 95\%: 1.13-1.85), y además los autores sugieren que el perfil de riesgo-beneficio no respalda una recomendación del uso habitual de warfarina para la reducción del accidente cerebrovascular en pacientes en hemodiálisis (35). En un metaanálisis de 2007 se encontró que aquellos con ERC terminal tratados con warfarina tienen un riesgo aumentado en 10 veces de presentar un sangrado mayor en comparación con la población general y que estos eventos se presentan inclusive cuando el INR está entre 1.5 -2.0 (36). En nuestro estudio, de los 107 pacientes con warfarina, 10 sangraron (9.34\%); de estos solo dos usaban ASA y no todos estaban sobreanticoagulados al momento del sangrado. Se evidenció una tasa de sangrado menor de $5.6 \%$ y de sangrado mayor de $3.7 \%$, la cual es inferior al estudio de Kooiman et al (2014), donde reporta $15.6 \%$ para este último tipo de sangrado teniendo en cuenta que en este estudio su muestra fue mayor ( $n=724)$ (37). Dicho estudio también reportó sangrados en pacientes con INR en rango terapéutico, lo cual se muestra en nuestro estudio (dos pacientes con sangrado mayor y uno con sangrado menor).

A pesar de la mayor seguridad demostrada con el uso de DOAC, en este estudio se encontró que la warfarina fue el 
Juliana Marcela Bustamante-Trespalacios y cols.

Tabla 4. Descripción de los pacientes que presentaron hemorragias.

\begin{tabular}{|c|c|c|c|c|c|c|c|c|c|c|c|c|}
\hline$\#$ & $\begin{array}{l}\text { Tipo de } \\
\text { sangrado }\end{array}$ & Sexo & $\begin{array}{c}\text { Edad } \\
\text { (años) }\end{array}$ & $\begin{array}{l}\text { Régimen } \\
\text { de salud }\end{array}$ & Fatal & $\begin{array}{c}\text { Sitio del } \\
\text { sangrado }\end{array}$ & $\begin{array}{c}\text { TFG ml/ } \\
\mathrm{min} / 1.73 \mathrm{~m} 2\end{array}$ & Anticoagulante & INR & Transfusión & \begin{tabular}{c|} 
Causa de \\
anticoagulación
\end{tabular} & Comorbilidades \\
\hline 1 & Menor & $\mathrm{H}$ & 87 & $\mathrm{C}$ & No & Gastrointestinal & 40.10 & Warfarina & 3.2 & No & FA no valvular & $\begin{array}{l}\text { Enfermedad pulmonar, } \\
\text { HTA }\end{array}$ \\
\hline 2 & Menor & $\mathrm{H}$ & 80 & $\mathrm{C}$ & No & Gastrointestinal & 58 & Warfarina & 3.2 & No & FA no valvular & $\begin{array}{l}\text { HTA, enfermedad } \\
\text { pulmonar, hipotiroidismo, } \\
\text { falla cardiaca. }\end{array}$ \\
\hline 3 & Menor & $\mathrm{H}$ & 85 & $\mathrm{C}$ & No & Tejido Blando & 56.90 & Dabigatrán & NA & No & FA no valvular & $\begin{array}{l}\text { HTA, dislipidemia, falla } \\
\text { cardíaca, Hipotirodismo, } \\
\text { demencia. }\end{array}$ \\
\hline 4 & Menor & $\mathrm{H}$ & 77 & $\mathrm{C}$ & No & Genitourinario & 33.30 & Apixabán & NA & No & FA no valvular & $\begin{array}{l}\text { LES, hipotiroidismo, HTA, } \\
\text { enfermedad pulmonar. }\end{array}$ \\
\hline 5 & Menor & $\mathrm{H}$ & 94 & $\mathrm{C}$ & No & Genitourinario & 21.60 & Dabigatrán y ASA & NA & No & FA no valvular & $\begin{array}{l}\text { ACV, falla cardiaca, DM2, } \\
\text { HTA, hipotiroidismo, } \\
\text { hiperplasia prostática }\end{array}$ \\
\hline 6 & Menor & $\mathrm{H}$ & 71 & $\mathrm{C}$ & No & Tejido blando & 46.00 & Warfarina & 2.5 & No & $\begin{array}{l}\text { FA no valvular } \\
\text { y trombo } \\
\text { intramural }\end{array}$ & $\begin{array}{l}\text { Falla cardiaca con } \\
\text { cardiodesfibrilador } \\
\text { implantable }\end{array}$ \\
\hline 7 & Menor & M & 87 & $\mathrm{C}$ & No & Tejido blando & 51.00 & Dabigatrán & NA & No & $\begin{array}{l}\text { FA no valvular y } \\
\text { TEP crónico }\end{array}$ & Enfermedad pulmonar \\
\hline 8 & Menor & $\mathrm{H}$ & 94 & $\mathrm{C}$ & No & Tejido blando & 40.70 & Rivaroxabán & NA & No & FA no valvular & $\begin{array}{l}\text { HTA, falla cardiaca, } \\
\text { enfermedad pulmonar }\end{array}$ \\
\hline 9 & Menor & M & 73 & $\mathrm{C}$ & No & Gastrointestinal & 55.80 & Apixabán & NA & No & ETEV & $\begin{array}{l}\text { HTA dislipidemia, } \\
\text { osteoporosis, enfermedad } \\
\text { pulmonar }\end{array}$ \\
\hline 10 & Menor & $\mathrm{H}$ & 53 & $\mathrm{C}$ & No & Genitourinario & TRR & Rivaroxabán & NA & No & ETEV & $\begin{array}{l}\text { TRM,vejiga neurogénica y } \\
\text { sonda vesical, HTA, DM2, } \\
\text { epilepsia. }\end{array}$ \\
\hline 11 & Menor & $\mathrm{H}$ & 54 & S & No & $\begin{array}{l}\text { Tejido blando } \\
\text { Mucosa nasal }\end{array}$ & 22.30 & Warfarina & 4.3 & No & FA no valvular & $\begin{array}{l}\text { HTA, falla cardiaca con } \\
\text { cardioresincronizador, } \\
\text { cirrosis hepática }\end{array}$ \\
\hline 12 & Menor & $\mathrm{H}$ & 79 & $\mathrm{~S}$ & No & $\begin{array}{l}\text { Tejido blando } \\
\text { Mucosa oral }\end{array}$ & 45.90 & Warfarina & 8.8 & No & FA no valvular & HTA, DM2 \\
\hline 13 & Menor & $\mathrm{H}$ & 87 & S & No & Mucosa nasal & 38.20 & Warfarina & 6.6 & No & FA no valvular & $\begin{array}{l}\text { HTA, portador de } \\
\text { marcapaso bicameral por } \\
\text { bloqueo atrioventricular, } \\
\text { falla cardiaca. }\end{array}$ \\
\hline 14 & Mayor & $\mathrm{H}$ & 68 & S & No & $\begin{array}{l}\text { Gastrointestinal } \\
\text { Mucosa nasal }\end{array}$ & 55.70 & Warfarina & 8.2 & $\mathrm{Si}$ & $\begin{array}{l}\text { FA no valvular/ } \\
\text { flutter }\end{array}$ & $\begin{array}{l}\text { Falla cardiaca, enfermedad } \\
\text { pulmonar, HTA }\end{array}$ \\
\hline 15 & Mayor & $\mathrm{H}$ & 54 & $\mathrm{~S}$ & No & Gastrointestinal & TRR & Warfarina & 1.73 & $\mathrm{Si}$ & $\begin{array}{l}\text { FA no valvular y } \\
\text { trombo auricular }\end{array}$ & $\begin{array}{l}\text { HTA, sepsis de origen en } \\
\text { tejidos blandos recurrente } \\
\text { por fistula arteriovenosa, } \\
\text { úlcera péptica }\end{array}$ \\
\hline 16 & Mayor & M & 82 & S & No & Gastrointestinal & 59.00 & Rivaroxabán & NA & No & FA no valvular & HTA, falla cardíaca \\
\hline 17 & Mayor & $\mathrm{H}$ & 95 & $\mathrm{C}$ & SI & \begin{tabular}{|l|} 
Sistema \\
nervioso central \\
(trauma)
\end{tabular} & 28.06 & Apixabán & NA & No & FA no valvular & $\begin{array}{l}\text { Falla cardiaca y } \\
\text { enfermedad coronaria, } \\
\text { enfermedad pulmonar, } \\
\text { enfermedad diverticular e } \\
\text { hipotiroidismo. }\end{array}$ \\
\hline 18 & Mayor & M & 79 & $\mathrm{C}$ & No & Gastrointestinal & 28.40 & Warfarina & 2.2 & No & FA no valvular & $\begin{array}{l}\text { Falla cardiaca, enfermedad } \\
\text { pulmonar, HTA, } \\
\text { hipotiroidismo, enfermedad } \\
\text { diverticular y úlcera } \\
\text { gástrica. }\end{array}$ \\
\hline 19 & Mayor & $\mathrm{H}$ & 82 & $\mathrm{C}$ & No & Gastrointestinal & 59.00 & Rivaroxabán & NA & $\mathrm{Si}$ & FA no valvular & $\begin{array}{l}\text { HTA, falla cardiaca, } \\
\text { cardioresincronizador, } \\
\text { enfermedad pulmonar }\end{array}$ \\
\hline 20 & Mayor & $\mathrm{H}$ & 68 & $\mathrm{C}$ & No & Genitourinario & 49.60 & Warfarina & 2.43 & No & $\begin{array}{l}\text { Válvula } \\
\text { mecánica aórtica }\end{array}$ & $\begin{array}{l}\text { ACV con vejiga } \\
\text { neurogénica, hiperplasia } \\
\text { prostática. HTA, } \\
\text { enfermedad pulmonar. }\end{array}$ \\
\hline
\end{tabular}


medicamento más usado (45.0\%), aun cuando $65.5 \%$ de los pacientes pertenecían al régimen contributivo, lo que podría conducir a cuestionarse el motivo de elección de la warfarina sobre los demás anticoagulantes y considerar si realmente el peso de la evidencia en la población colombiana es suficiente para elegir el tipo de anticoagulante o si los factores sociales, económicos y culturales tienen mayor peso al momento de la prescripción de estos.

Un desenlace importante para los pacientes con uso de warfarina, es el TRT, el cual estima el porcentaje de tiempo en que el INR está dentro del rango de tratamiento deseado y es ampliamente utilizado como un indicador de control de anticoagulación. (38). Este estudio encontró que el TRT era $48.8 \%$, lo que significa que en más de la mitad del tiempo los pacientes se encontraban por fuera del objetivo. Esta falta de meta está bien descrita en la literatura como por Chaaban et al (2015), quienes presentaron datos sobre el uso de warfarina en pacientes en hemodiálisis (HD) versus aquellos con función renal normal y encontró que no sólo los pacientes en HD presentaban más hemorragias, sino que el INR estaba en rango terapéutico solo durante el $25 \%$ del tiempo para este grupo (39). Yang et al, (2017), recientemente publicó su estudio donde encontró que solo $21 \%$ de los pacientes en diálisis tenían un TRT mayor a $60 \%$; además que durante el $30 \%$ del tiempo el INR estaba notablemente fuera de rango (INR menor de 1.5 o mayor de 3. 5) (40). Todo esto hace realmente impredecible la anticoagulación con warfarina y abre la posibilidad de desarrollar más estudios al respecto dado que es una población diferente, posiblemente de edad avanzada, con enfermedades graves asociadas y con dificultades para el acceso al sistema de salud y adherencia al tratamiento.

En cuanto a los DOAC, el apixabán está aprobado a dosis de $5 \mathrm{mg}$ dos veces al día y con necesidad de ajuste a $2.5 \mathrm{mg}$ cada 12 horas para los pacientes que cumplen dos de los siguientes criterios: creatinina mayor a $1.5 \mathrm{mg} / \mathrm{dL}$, mayores de 80 años o peso inferior a $60 \mathrm{~kg}$ de acuerdo con estudio ARISTOTLE (41). Éste excluyó a los pacientes con TFG menor de $25 \mathrm{~mL} / \mathrm{min}$, por lo cual no existe buena representación de la población en estadios 4 y 5 de ERC. A pesar de esto la actualización de las guías AHA 2019 de fibrilación auricular y la FDA recomiendan usar el apixabán para pacientes con ERC avanzada inclusive en hemodiálisis (42). Esto sigue siendo motivo de controversia pues la recomendación se basa en un estudio de farmacodinamia con 16 pacientes y un registro del MEDICARE de USA con lo cual la calidad de la evidencia científica no es la mejor (43, 44). En 2019 se presentaron resultados preliminares del estudio RENAL-AF que comparó apixabán vs warfarina en diálisis, éste no fue concluyente dado que no logró el reclutamiento esperado por lo cual se suspendió antes del tiempo estipulado, además el TRT para los pacientes tratados con warfarina fue $44 \%$ con una gran proporción de pacientes en rango subterapéutico (45). Nuestro estudio muestra que de 34 pacientes con uso de apixabán, tres presentaron sangrado (tasa de 8.8\%); uno de ellos fue el único caso de hemorragia fatal, sin embargo, se asoció a TEC el cual causó la muerte, este paciente tenía edad avanzada y una TFG $28 \mathrm{~mL} / \mathrm{min}$ por lo cual tenía dosis del medicamento ajustada.

De igual forma, el rivaroxabán en su estudio principal (ROCKET-FA) excluyó TFG $<30 \mathrm{~mL} / \mathrm{min}$ (46). Existen estudios observacionales y farmacológicos que evidencian la seguridad del rivaroxabán en el estadio G4 de ERC por lo cual en Estados Unidos está aprobado para dicha indicación, mientras que otros países como Canadá sólo utilizan su recomendación tradicional $(47,48)$. En nuestro estudio sangraron 4 de 75 sujetos con rivaroxabán (tasa de sangrado de 5.3\%), y de ellos todos estaban en estadio 3 de ERC, excepto uno que requirió terapia de reemplazo renal transitorio.

El dabigatrán está contraindicado para pacientes con TFG $<15 \mathrm{~mL} / \mathrm{min}$ según lineamientos internacionales, pero ni siquiera para el estadio 4 de ERC existen estudios con grandes cantidades de pacientes. El nuestro, muestra que la tasa más alta de sangrado se dio con este medicamento (37.5\%), por lo que, teniendo presente su alta tasa de eliminación renal, consideramos que no es de elección en pacientes con ERC (49), aun así, tiene aprobación de la FDA para usar dosis ajustada de $75 \mathrm{mg}$ cada 12 horas basado en un modelo de predicción farmacológica. (50)

La eficacia de los anticoagulantes orales está muy bien establecida en estudios controlados y se requiere más información sobre la seguridad de los mismos en las diferentes poblaciones. En este estudio se describieron los perfiles clínicos y los eventos hemorrágicos de los sujetos anticoagulados durante el seguimiento hecho por la IPS Universitaria Universidad de Antioqia desde enero de 2017 a diciembre de 2018. Este estudio representa la cohorte más grande publicada hasta la fecha, de pacientes con enfermedad renal crónica y uso de anticoagulantes (tanto warfarina como DOAC) en Colombia y en Latinoamérica.

Las limitaciones del estudio actual están relacionadas con la naturaleza retrospectiva del estudio, la recolección de los datos y la falta de algunos de ellos. El cálculo de la tasa de filtración glomerular por la ecuación CKD-EPI tiene limitaciones teniendo en cuenta que la raza no estaba registrada en la gran mayoría de los pacientes. La población en estadio G5 de ERC fue escasa. No se tuvo en cuenta el CHADs2Vasc ni el HAS-BLED para la interpretación de los resultados para los pacientes con fibrilación auricular.

\section{Conclusión}

La causa de anticoagulación más común fue la fibrilación auricular y el estadio de enfermedad renal más frecuente fue el G3. En forma global, se apreció una baja tasa de sangrado mayor, lo que podría estar relacionado con el estrecho seguimiento de una clínica de anticoagulación, este tipo de sangrado fue más frecuente en los pacientes con warfarina lo cual se asoció a un TRT bajo (48.8\%). Los medicamentos más seguros fueron el apixabán y el rivaroxabán con dosis ajustadas. 
La cantidad de pacientes en estadio G5 de ERC fue muy baja por lo cual no es posible sacar conclusiones sobre este grupo en particular.

Se requiere de estudios clínicos aleatorizados para definir la estrategia de anticoagulación más segura en esta población.

\section{Agradecimientos}

A todo el personal de salud, administrativo e investigación de la clínica de anticoagulación de la IPS Universitaria Universidad de Antioqia por la disponibilidad de la información y la recolección de los datos.

\section{Referencias}

1. Turagam MK1, Parikh V2, Afzal MR2, et al. Replacing warfarin with a novel oral anticoagulant: Risk of recurrent bleeding and stroke in patients with warfarin ineligible or failure in patients with atrial fibrillation (The ROAR study). $J$ Cardiovasc Electrophysiol. 2017; 28(8):853-861

2. Munir Pirmohamed,M. Warfarin: almost 60 years old and still causing problems. Br J Clin Pharmacol. 2006; 62(5): 509-511.

3. Kumar S, De Lusignan S, McGovern A, Correa A, Hriskova M, Gatenby P, et al. Ischaemic stroke, haemorrhage, and mortality in older patients with chronic kidney disease newly started on anticoagulation for atrial fibrillation: A population based study from UK primary care. BMJ. 2018;360:1-10.

4. Jaramillo Salamanca RG, Jiménez Gómez JD, Pinilla Roa AE. Uso de los anticoagulantes orales directos en la práctica clínica. Rev Fac Med.2016;64(2):295.

5. Harel Z, Sholzberg M, Shah PS, Pavenski K, Harel S, Wald R, et al. Comparisons between novel oral anticoagulants and vitamin $\mathrm{K}$ antagonists in patients with CKD. J Am Soc Nephrol. 2014;25(3):431-42.

6. Harel Z, Sood MM, Perl J. Comparison of novel oral anticoagulants versus vitamin K antagonists in patients with chronic kidney disease. Curr Opin Nephrol Hypertens. 2015;24(2):183-92.

7. Meena Zareh, BS,Andrew Davis, BS,and Sean Henderson. Reversal of Warfarin-Induced Hemorrhage in the Emergency Department. West J Emerg Med. 2011; 12(4): 386-392.

8. Laverde, LP. Gómez, SE.Montenegro,AC. et al. Experiencia de una clínica de anticoagulación. Rev Colomb Cardiol 2015;22(5):224-230

9. Lega J-C, Bertoletti L, Gremillet C, Boissier C, Mismetti P, Laporte S. Consistency of safety profile of new oral anticoagulants in patients with renal failure. J Thromb Haemost 2014; 12: 337-43.

10. Kimachi M, Furukawa TA, Kimachi K, Goto Y, Fukuma S, Fukuhara S. Direct oral anticoagulants versus warfarin for preventing stroke and systemic embolic events among atrial fibrillation patients with chronic kidney disease. Cochrane Database of Systematic Reviews 2017; 11. Art. No.: CD011373.

11. Padrini R. Clinical Pharmacokinetics and Pharmacodynamics of Direct Oral Anticoagulants in Patients with Renal Failure. Eur J Drug Metab Pharmacokinet. 2019;44(1).

12.Parker, K. Mitra, S. Tachi J. Is anticoagulating haemodialysis patients with non-valvular atrial fibrillation too risky?. British Journal of Haematology. 2018; 181: 725-736

13. Kimachi M, Furukawa TA, Kimachi K, Goto Y, Fukuhara S. New oral anticoagulants versus warfarin for preventing stroke and systemic embolic events among atrial fibrillation patients with chronic kidney disease. Cochrane Database Syst Rev. 2014;2014(11).

14. Ramagopalan S V., Stamp E, Sammon CJ, Besford M, Carroll R, Mehmud F, et al. Renal function and oral anticoagulant treatment of incident non-valvular atrial fibrillation: A retrospective study. Future Cardiol. 2019;15(4):301-9.

15. Sardar P, Chatterjee S, Herzog E, Nairooz R, Mukherjee D, Halperin JL. Novel oral anticoagulants in patients with renal insufficiency: A meta-analysis of randomized trials. Can J Cardiol [Internet]. 2014;30(8):888-97.

16. R. Marinigh, D.A. Lane, G.Y.H. Lip, Severe renal impairment and stroke prevention in atrial fibrillation, J. Am. Coll. Cardiol 2011; 57:1339-1348.

17. Providencia R, Marijon E, Boveda S, Barra S, Narayanan K, Le Heuzey JY, Gersh BJ, Goncalves L. Meta-analysis of the influence of chronic kidney disease on the risk of thromboembolism among patients with nonvalvular atrial fibrillation. Am J Cardiol. 2014;114(4):646-53.

18. Hijazi Z., Hohnloser S.H., Oldgren J., et al. Efficacy and safety of dabigatran compared with warfarin in relation to baseline renal function in patients with atrial fibrillation: a RE-LY (Randomized Evaluation of Long-term Anticoagulation Therapy) trial analysis. Circulation 2014 129: 961-970.
19. Manesh R. Patel, et al. Rivaroxaban versus Warfarin in Nonvalvular Atrial Fibrillation. N Engl J Med 2011; 365:883-891. DOI: 10.1056/NEJMoa1009638

20. Granger, C. B. Apixaban versus warfarin in patients with atrial fibrillation. New Eng J Med 2011; 365, 981-992. DOI: 10.1056/NEJMoa1107039

21. Robert P. Giugliano, et al. Edoxaban versus Warfarin in Patients with Atrial Fibrillation. N Engl J Med 2013; 369: 2093-2104

22. Lega JC, Bertoletti L, Gremillet C, Boissier C, Mismetti P, Laporte S. Consistency of safety profile of new oral anticoagulants in patients with renal failure. J Thromb Haemost. 2014;12(3):337-43.

23. Shin JI, Secora A, Caleb Alexander G, Inker LA, Coresh J, Chang AR, et al. Risks and benefits of direct oral anticoagulants across the spectrum of GFR among incident and prevalent patients with atrial fibrillation. Clin J Am Soc Nephrol. 2018;13(8):1144-52.

24. Hughes S, Szeki I, Nash MJ, Thachil J. Anticoagulation in chronic kidney disease patients - The practical aspects. Clin Kidney J. 2014;7(5):442-9.

25. Kidney Disease: Improving Global Outcomes (KDIGO) Blood Pressure Work Group. KDIGO clinical practice guideline for the management of blood pressure in chronic kidney disease. Kidney Int Suppl 2012;2:337-414.

26. Schulman S, Kearon C. Subcommittee on Control of Anticoagulation of the Scientific and Standardization Committee of the International Society on Thrombosis and Haemostasis. Definition of major bleeding in clinical investigations of antihemostatic medicinal products in non-surgical patients. J Thromb Haemost 2005; 3: 692-4.

27. Tan et al. Warfarin use and stroke, bleeding and mortality risk in patients with end stage renal disease and atrial fibrillation: a systematic review and meta-analysis. BMC Nephrology (2016) 17:157. DOI:10.1186/s12882-016-0368-6

28. Cuenta de alto costo. Situación de la enfermedad renal crónica, hipertensión arterial y diabetes mellitus en Colombia. (Internet) (Consultado 2017 Dic 12) en Disponible en: https://cuentadealtocosto.org/site/images/Situaci\%C3\%B3n_de_la_Enfermedad_Renal_Cr\%C3\%B3nica_en_Colombia_2015.pdf.

29. Wizemann V, Tong L, Satayathum S, et al. Atrial fibrillation in hemodialysis patients: clinical features and associations with anticoagulant therapy. Kidney International 2010; 77 (12):1098 - 1106. DOI: 10.1038/ki.2009.477

30. Soliman EZ, Prineas RJ, Go AS, et al. Chronic kidney disease and prevalent atrial fibrillation: the Chronic Renal Insufficiency Cohort (CRIC). Am Heart J 2010;159:1102-7.

31.R.Marinigh, D.A.Lane, G.Y.H.Lip, Severe renal impairment and stroke prevention in atrial fibrillation, J.Am. Coll.Cardiol 2011; 57:1339-1348. DOI:10.1016/j. jacc.2010.12.013.

32. Providencia R, Marijon E, Boveda S, Barra S, Narayanan K, Le Heuzey JY, Gersh BJ, Goncalves L. Meta-analysis of the influence of chronic kidney disease on the risk of thromboembolism among patients with nonvalvular atrial fibrillation. Am J Cardiol. 2014;114(4):646-53. DOI:10.1016/j.amjcard.2014.05.048

33. Makani A, Saba S, Jain SK, Bhonsale A, Sharbaugh MS, Thoma F, et al. Safety and Efficacy of Direct Oral Anticoagulants Versus Warfarin in Patients With Chronic Kidney Disease and Atrial Fibrillation. Am J Cardiol [Internet]. 2020;125(2):210-4.Available from: https://doi.org/10.1016/j.amjcard.2019.10.033

34. Feldberg J, Patel P, Farrell A, Sivarajahkumar S, Cameron K, Ma J, et al. A systematic review of direct oral anticoagulant use in chronic kidney disease and dialysis patients with atrial fibrillation. Nephrol Dial Transplant. 2019;34(2):265-77.

35.Shah, M., Tsadok, M.A., Jackevicius, C.A., Essebag,V., Eisenberg, M.J., Rahme, E., Humphries, K.H., Tu, J.V., Behlouli, H., Guo, H. \& Pilote, L. Warfarin use and the risk for stroke and bleeding in patients with atrial fibrillation undergoing dialysis. Circulation 2014; 129,1193-1203.

36. Elliott, M.J., Zimmerman, D. \& Holden, R.M. Warfarin anticoagulation in hemodialysis patients: a systematic review of bleeding rates. American Journal of Kidney Diseases 2007; 50, 433-440. DOI: 10.1053/j.ajkd.2007.06.017

37. Kooiman J, van Rein N, Spaans B, van Beers KA, Bank JR, et al. Efficacy and safety of vitamin K-antagonists (VKA) for atrial fibrillation in non-dialysis dependent chronic kidney disease. PLoS One. 2014; 9;9(5):e94420.

38. Miranda, H. Osorio, S. Giraldo, DP, et al. Tiempo en rango terapéutico (TRT) en clínica de anticoagulación, Reportes de eventos adversos y factores asociados a bajo TRT. Acta Médica Colombiana, 2016; 41(1)

39. Chaaban, A., Gebran, N., Abdelmegid, K., Abouchacra, S., Soliman, K., Agha, L. y Butt, L. Effectiveness and safety of warfarin use for cardiac indications in hemodialysis patients. Nephrology Dialysis Transplant 2015; 30, 563-564.

40. Yang, F., Hellyer, J.A., Than, C., Ullal, A.J., Kaiser, D.W., Heidenreich, P.A., Hoang, D.D., Winkelmayer, W.C., Schmitt, S., Frayne, S.M., Phibbs, C.S. y Turakhia, M.P. Warfarin utilization and anticoagulation control in patients with atrial fibrillation and chronic kidney disease. Heart 2017; 103, 818-826. DOI: 10.1136/heartjnl-2016-309266

41. Granger, C. B. Apixaban versus warfarin in patients with atrial fibrillation. New England Journal of Medicine 2011;365,981-992. DOI: 10.1056/NEJMoa1107039 
42. Society.January CT, et al. 2019 AHA/ACC/HRS Focused Update of the 2014 AHA/ACC/HRS Guideline for the Management of Patients With Atrial Fibrillation: A Report of the American College of Cardiology/American Heart Association Task Force on Clinical Practice Guidelines and the Heart Rhythm. J Am Coll Cardiol. 2019 Jan 21.

43. Wang, X., Tirucherai, G., Marbury, T., Wang, T., Chang, M., Zhang, D., Song, Y., Pursley, J., Boyd, R. y Frost, R. Pharmacokinetics, pharmacodynamics, and safety of apixaban in subjects with end stage renal disease on hemodialysis. The journal of clinical Pharmacology, 2016: 56 (5), 628-636. DOI:10.1002/jcph.628

44. Siontis KC1,2, Zhang X3, Eckard A4, Bhave N1, et al. Outcomes Associated With Apixaban Use in Patients With End-Stage Kidney Disease and Atrial Fibrillation in the United States, Circulation. 2018;138(15):1519-1529.

45. RENal hemodialysis patients ALlocated apixaban versus warfarin in Atrial Fibrillation (RENAL-AF) Randomized Clinical Trial. AHA Congress 2019 (https:// www.acc.org/latest-in-cardiology/clinical-trials/2019/11/15/17/29/renal-af).
46. Manesh R. Patel, et al. Rivaroxaban versus Warfarin in Nonvalvular Atrial Fibrillation. N Engl J Med 2011; 365:883-891. DOI: 10.1056/NEJMoa1009638

47. Langas M, Moore K T. Renal Impairment and Chronic Hemodialysis on the pharmacokinetics, pharmacodynamics and Safety of Rivaroxaban. Journal of Renal Medicine 2017; 1:2-9. DOI: http://www.imedpub.com/renal-medicine/

48. Chan, K.E., Edelman, E.R., Wenger, J.B. et al. Dabigatran and Rivaroxaban Use in Atrial Fibrillation Patients on Hemodialysis. Circulation 2015; 131(11): 972-979. DOI: 10.1161/CIRCULATIONAHA.114.014113.

49. Knauf, F., Chaknos, M., Berns, J.S., Perazella, M.A. Dabigatran and Kidney Disease: A Bad Combination. Clin J Am Soc Nephrol 2013 8:1591-1597. DOI: 10.2215/CJN.01260213

50. Kooiman, J., Van der Hulle, T., Maas, H., Wiebe, S., Formella, S., et al. Pharmacokinetics and Pharmacodynamics of Dabigatran $75 \mathrm{mg}$ b.i.d. in Patients With Severe Chronic Kidney Disease. Journal of the American College of Cardiology. May 2016; 67 (20) 2442-2444; DOI: 10.1016/j.jacc.2016.03.516 